Itinéraires Itinéraires

Littérature, textes, cultures

2012-2 | 2012

Intime et politique

\title{
Intimité et discours politique : l'intime dans les campagnes électorales de 1848
}

\section{Marieke Stein}

\section{(2) OpenEdition}

1 Journals

Édition électronique

URL : http://journals.openedition.org/itineraires/1087

DOI : $10.4000 /$ itineraires. 1087

ISSN : 2427-920X

Éditeur

Pléiade

\section{Édition imprimée}

Date de publication : 1 novembre 2012

Pagination : 35-46

ISBN : 978-2-336-00027-5

ISSN : $2100-1340$

\section{Référence électronique}

Marieke Stein, «Intimité et discours politique : l'intime dans les campagnes électorales de 1848 », Itinéraires [En ligne], 2012-2 | 2012, mis en ligne le 01 novembre 2012, consulté le 10 décembre 2020 URL : http://journals.openedition.org/itineraires/1087 ; DOI : https://doi.org/10.4000/itineraires. 1087

\section{cc)}

Itinéraires est mis à disposition selon les termes de la licence Creative Commons Attribution - Pas d'Utilisation Commerciale - Pas de Modification 4.0 International. 


\section{Intimité et discours politique : l'intime dans les campagnes électorales de 1848}

\section{Abstract}

Since the 1950 American election campaigns (from Eisenhower onwards), political marketing has brought to the foreground the family life of the candidates, their love lives, emotions... anything related to their personal lives. In France, a similar trend was noted during the 2002 election, which was even more apparent in 2007. But what was the situation in 1848, at the first French elections with universal suffrage? Did the candidates for the national parliament base their electoral strategy on their personal lives or on their political vision?

This study examines the political statements of authors who stood for election in 1848: Hugo, Lamartine, Dumas, Sue, Vigny, etc. These authors, associated with the Romanticism Movement in 1848, all have an unusual relationship with the Self. Moreover, Romanticism closely links the Self and the political: therefore, the relatively impersonal tone of their statements is all the more surprising.

The main purpose of this article is to better comprehend the role of the self in political life from a historical perspective.

Keywords : election campaigns, universal suffrage, romanticism, authors in politic, Self, electoral statements

Mots clés : élections, 1848, professions de foi, romantisme, intime, écrivains en politique

Les campagnes électorales sont les moments de la vie politique où s'observe actuellement le mieux le lien entre l'intime et le politique. Depuis les années 1950 aux États-Unis (voir la campagne d'Eisenhower en 1952, celle de Kennedy quelques années plus tard), la communication politique a mis sur le devant de la scène, dans les campagnes électorales, la vie familiale, amoureuse, les douleurs personnelles, les «blessures" d'enfance des candidats. En France, en 2002 et plus encore en 2007, on a pu constater à quel point l'intime prenait de l'importance lors des campagnes présidentielles, tendance favorisée par la télévision et, plus récemment, par Internet, média apte à transmettre des états d'âme et à 
jouer la connivence avec l'électeur potentiel, notamment par l'intermédiaire des blogs de personnalités politiques.

Si les chercheurs, depuis plusieurs années déjà, pointent cet envahissement de la scène politique par ce qui ressortit traditionnellement au domaine de l'intériorité, du " gardé pour soi », de ce qui doit échapper au jugement du collectif et à la compétence sociale ${ }^{1}$, qu'en était-il au XIX ${ }^{\mathrm{e}}$ siècle, à l'heure où les citoyens faisaient leur apprentissage de la vie politique, et où les candidats en étaient seulement à découvrir et inventer leurs modes de campagne? Quelle est la place de l'intime dans ces premières campagnes électorales où se fait l'apprentissage de la démocratie - mais aussi des stratégies de parole? Les élections à l'Assemblée constituante de 1848 semblent un terrain intéressant à explorer dans cette perspective. En effet, les élections législatives de 1848 sont les premières élections au suffrage universel, et elles mobilisent l'ensemble de la population masculine majeure. La plupart de ces citoyens n'ont jamais voté, et un grand nombre d'entre eux sont incultes, voire illettrés. Des déductions hâtives pourraient nous amener à penser qu'un discours « sensible », fondé sur la personnalité du candidat, ses émotions, sa vie personnelle, aurait dû être plus efficace sur ces masses qu'un discours rationnel, argumenté. Qu'un discours fondé sur l'ethos et le pathos - les deux catégories de la rhétorique classique qui ont le plus à voir avec l'intime - toucherait davantage les masses que des raisonnements et des arguments politiques (le logos). Lorsque l'on songe également que le seul vecteur de la parole politique est alors l'imprimé, et que les articles de presse comme les brochures et les affiches proposent des dissertations politiques denses et d'aspect rébarbatif, on se dit que sans doute, les candidats désireux de parler au peuple, pour se distinguer et susciter la sympathie, avaient intérêt à parler d'eux-mêmes, à dévoiler des pans de leur personnalité, de leur vie privée, de tout «ce qu'il y a de plus intérieur », pour reprendre l'emploi augustinien du terme intime ${ }^{2}$. Mais c'est là, nous le verrons, une conception moderne, totalement étrangère au premier âge de la République.

\section{Le romantisme : un double mouvement en soi et vers le monde}

Pour évaluer la place de l'intime dans les professions de foi des candidats aux premières élections réellement démocratiques, nous avons étudié

1. Michaël Fœssel définit l'intime comme ce que l'individu retranche du domaine de la visibilité commune, se ménageant ainsi une distance et une liberté fondamentales par rapport au collectif - et au politique. Voir Michaël Fœssel, La Privation de l'intime. Mises en scènes politiques des sentiments, Paris, Seuil, 2008, p. 17. Définition proche de celle de Lila Ibrahim-Lamrous et Séveryne Muller : «D’un point de vue psychosociologique, l'intimité se caractérise par le désir de se soustraire au corps social [...]». Lila IbrahimLamrous et Séveryne Muller, L'Intimité, Clermont-Ferrand, Presses Universitaires BlaisePascal, 2005, p. 8.

2. Saint-Augustin, Confessions, III, $6:$ « tu autem eras interior intimo meo ». 
les proclamations électorales et autres déclarations de foi des principaux écrivains candidats aux élections de 1848, qu'il s'agisse des élections à l'Assemblée constituante du 23 avril 1848, des élections complémentaires lors desquelles fut élu notamment Victor Hugo (4 et 5 juin), ou de l'élection présidentielle qui valut un cuisant échec à Lamartine (10 décembre 1848). Alexandre Dumas (candidat malheureux de l'Yonne), Balzac, (une vingtaine de voix seulement, à Paris!), Eugène Sue (battu en 1848 dans le Loiret, mais élu aux élections complémentaires de 1850 à Paris), Alfred de Vigny (60 voix charentaises seulement en 1848, guère plus en 1849), Béranger (élu à Paris mais rapidement démissionnaire), Lamennais (député de la Seine durant toute la Deuxième République) ou des écrivains moins connus comme Lepoitevin Saint-Alme (élu), le poète Méry, Arsène Houssaye ou encore le dramaturge Latour de Saint-Ybars (non élus) ont tous profité de «l'illusion lyrique » du printemps 1848 ainsi que de leur foi - et de la foi collective, d'inspiration saint-simonienne - dans la mission sociale des poètes pour tenter de devenir enfin les guides du peuple que décrit Paul Bénichou dans sa monumentale histoire de la vision du monde des écrivains romantiques $^{3}$. Jamais élection ne vit tant de candidatures d'hommes de lettres... Pourquoi étudier les professions de foi électorales des écrivains? Ce choix s'explique en partie par la quantité d'écrits qu'ils ont laissés, et par la comparaison aisée qu'on peut faire entre leurs écrits politiques et publics, d'une part, et, d'autre part, leurs écrits littéraires (eux aussi publics) et privés (correspondances, journaux publiés souvent bien plus tard). De telles comparaisons mettent en lumière la place faite à l'intime dans les genres respectifs.

Surtout, les écrivains qui se présentent aux élections de 1848 sont proches du mouvement romantique, qui entend d'une manière spécifique le lien entre le moi et le monde, l'intimité et l'extériorité, l'individuel et l'universel. L'intimité est une composante essentielle du romantisme; d'ailleurs, Baudelaire définit en 1846 le romantisme par quatre tendances : «intimité, spiritualité, aspiration vers l'infini, couleur ${ }^{4} »$. Pour les écrivains romantiques, l'importance accordée au moi, le primat donné dans l'œuvre et dans la vie aux émotions, à la vie intérieure, découlent, certes, d'une désorientation voire d'un rejet du monde « extérieur » et de la vie publique, mais ils motivent en même temps un élan vers cette même universalité. L'intime des romantiques est à la fois le plus profond siège de l'individualité, - et se rapproche des notions de « sensibilité » ou de « sentiment »-, mais aussi le lieu de la rencontre avec l'autre et les autres. Comme l'explique Gérard Gengembre, après un premier mouvement de repli sur soi né d'un désaccord entre le moi et le monde, chez les romantiques « le sentiment tend

3. Voir Paul Bénichou, Le Sacre de l'écrivain, Le Temps des prophètes, Les Mages romantiques et L'École du désenchantement, republiés dans Romantismes, Paris, Gallimard, coll. « Quarto », 2004.

4. Charles Baudelaire, Salon de 1846, Paris, Garnier, 1962, p. 103. 
de plus en plus à se définir comme disposition sympathique à 1 'autre ${ }^{5}$ ». 1830 puis 1848 sont les deux grands moments de l'histoire où, enfin, les écrivains romantiques, même « mal en cour », peuvent prétendre se mettre au service du monde, et reconquérir l'accord perdu entre le moi et le monde - ou le peuple.

Le romantisme est alors caractérisé par un double mouvement d'introversion et d'extraversion, un élan dans et hors de soi. Les deux mouvements ne sont d'abord contradictoires qu'en apparence : c'est bien l'introspection, la vie « du dedans » qui donne à l'homme romantique la connaissance intime du vrai, et qui donc justifie, voire impose, son intervention dans la vie publique, laquelle ne consiste en définitive qu'à mettre cette connaissance du vrai au service des autres hommes. Ce mécanisme est en partie exposé par Maine de Biran dès 1816 :

\footnotetext{
C'est en nous-même qu'il faut descendre, c'est dans l'intimité même de la conscience qu'il faut habiter, pour jouir de la vérité et atteindre à la réalité de toutes choses. Par l'acte seul de la réflexion, par l'effort que fait l'homme qui s'arrache au monde extérieur pour s'étudier et se connaître, il se dispose à recevoir et à saisir le vrai ${ }^{6}$.
}

Ce qui explique que le poète romantique puisse devenir cette «figure générique, collective ${ }^{7}$ » apte à parler de tous les hommes en parlant de soi. C'est bien d'ailleurs au nom de ce savoir intime, qui est d'abord connaissance de soi, que Victor Hugo affirme se présenter aux élections complémentaires à la Constituante, lorsqu'il s'adresse aux cinq associations d'art et d'industrie : «Je suis résolu à toujours agir selon cette lumière qui est dans mon âme, et qui me montre le juste et le vrai ${ }^{8}$. " Chez Hugo, le domaine du poète est l'intériorité - très proche de l'intimité -, pensée comme profondeur, voire abîme. D'où le rapprochement fréquent, dans son œuvre, entre le poète qui cherche le vrai au fond de soi et au fond des choses, et le «plongeur». Il écrira d'ailleurs en 1872 : « Il est urgent que les législateurs prennent conseil des penseurs, que les hommes d'État, trop souvent superficiels, tiennent compte du profond travail des écrivains ${ }^{9}$. » On retrouve, dans ce réseau lexical hugolien de la profondeur, le sens étymologique d'intimus - qui n'est que le superlatif d'interior. C'est vraiment ce qu'il y a de plus profond en soi qui permet l'élan vers les hommes et vers le monde.

5. Gérard Gengembre, Le Romantisme, Paris, Ellipses, 1995, p. 10.

6. Maine de Biran, Journal, 25 novembre 1816, t. I, Neufchatel, La Baconnière, 1954, p. 240.

7. Gérard Gengembre, op. cit., p. 39.

8. Victor Hugo, « Séance des cinq associations », dans EEuvres complètes, vol. « Politique », éd. Jacques Seebacher (assisté de Guy Rosa), Paris, Laffont, 1995, p. 162.

9. Victor Hugo, Lettre à Léon Richer, 8 juillet 1872, dans Actes et Paroles III, Euvres complètes, Paris, Imprimerie nationale, p. 175 (nous soulignons). 


\title{
Les professions de foi des écrivains en 1848 : l'effacement du « je »
}

On pourrait donc s'attendre à ce que, hors même de toute stratégie persuasive, et en raison de leur conception du sacerdoce poétique et politique, les écrivains romantiques accordent une grande place à l'intime dans leurs déclarations de foi. Or il n'en est rien. La conception romantique de la mission des poètes, au lieu d'induire une propension à l'épanchement et à la représentation d'une intériorité, se manifeste au contraire par un souci accru d'objectivité, de réalisme, voire de dépersonnalisation du discours - sans doute pour désamorcer les préventions d'une partie de ce qu'on n'appelle pas encore la classe politique à l'encontre des écrivains « si tristement fourvoyé[s] dans la carrière politique ${ }^{10}$ ». En vertu de leur croyance en la rencontre du moi avec l'intériorité de la foule, certains écrivains romantiques ont même manifesté une certaine répugnance à faire campagne, que l'on constate en particulier chez Vigny (qui affirme ne pas faire campagne ${ }^{11}$ ), Lamartine et Hugo. Ce dernier, en 1848, refuse de se porter candidat, écrivant à Pierre Cauwet le 5 avril : « Je ne suis pas candidat, mais je ne suis pas refusant ${ }^{12}$. » De même Lamartine lors de l'élection présidentielle : « Je ne brigue pas les suffrages. Je ne les désire pas. Je ne fais ni manifeste ni programme ${ }^{13}$. » Pour lui comme pour d'autres poètes romantiques, les élections de 1848 doivent permettre l'élection spontanée, qui est de l'ordre de l'enthousiasme, du poète par le peuple, et elles doivent échapper aux tracasseries et aux bassesses de la séduction électorale. Dominique Dupart écrit justement :

\begin{abstract}
Voter en avril 1848, c'est encore manifester un enthousiasme irréductible aux trivialités de la politique. Le suffrage universel consacre naturellement une sensibilité d'inspiration humanitaire. Il offre au suffrage lyrique des poètes de faire entendre sa nature profonde, une harmonie entre le cour et l'âme - les sièges de l'inspiration lyrique -, et cette nouvelle démocratie d'opinion expérimentée par les masses ${ }^{14}$.
\end{abstract}

Croyant en ce que Dominique Dupart appelle le « suffrage lyrique », le poète romantique ne sent pas la nécessité de faire campagne, le sentiment

10. Selon une expression de L'Assemblée nationale, 17 janvier 1851, à propos de Victor Hugo.

11. «Je n'irai point, chers concitoyens, vous demander vos voix. Je ne reviendrai visiter au milieu de vous notre belle Charente qu'après que votre arrêt aura été rendu. » Alfred de Vigny, « Proclamation aux électeurs de la Charente », imprimerie de Guiraudet et Jouaust, 27 mars 1848.

12. Victor Hugo, Euvres complètes, édition chronologique publiée par J. Massin, Paris, CFL, 1967-1970, t. VII, p. 747.

13. Lamartine, Lettre aux journaux sur la présidence, dans Cuvres oratoires et écrits politiques, La France parlementaire (1834-1851), t. VI, 30 novembre 1848, Paris, Librairie internationale, 1864, p. 34.

14. Dominique Dupart, "Suffrage universel, suffrage lyrique chez Lamartine (18341848) », Romantisme, n 135, L'Élection au XIX siècle, 2007, p. 9-21, p. 10. 
naturel des foules devant spontanément désigner le poète, au gré du « surgissement unanime et soudain du sentiment chez le peuple, cet autre Moi de l'orateur populaire ${ }^{15} \gg$. Les élections de 1848 ont pourtant apporté un démenti à ces poètes: Vigny ne recueille que quelques dizaines de voix; si Victor Hugo remporte 59446 voix sans avoir fait campagne, la communion des âmes du poète et de son peuple ne suffit pas à lui donner un siège, et il faudra qu'il s'engage résolument dans la campagne pour être élu représentant de Paris aux élections complémentaires de juin. Quant à Lamartine, la sympathie réelle que lui voue le peuple ne résiste pas aux crises du printemps 1848 et les 17910 voix qu'il obtient à l'élection présidentielle - contre cinq millions et demi pour Louis-Napoléon Bonaparte! - montrent les limites du « suffrage lyrique ». Résultats qui ne font que conforter la méfiance des écrivains à l'égard de la pratique électorale - « censée encourager au mieux la médiocrité, au pire la corruption $^{16} \gg-$, voire le retrait de la plupart d'entre eux de la vie politique active après 1848 .

Qu'ils partagent ou non cet idéal de l'élection, les écrivains romantiques, dans leurs professions de foi, n'exposent pas davantage leur moi que d'autres candidats. Qu'il s'agisse de Dumas, de Hugo, d'Eugène Sue, leurs déclarations électorales reposent toujours sur un contenu exclusivement politique : on observe partout la même absence de toute considération individuelle, affective ou privée. Vigny expose largement dans sa proclamation Aux électeurs de la Charente « un projet politique, celui d'une république sage et modérée dont les valeurs fondamentales sont la propriété, le travail et la famille ${ }^{17} »$. Victor Hugo, dans sa proclamation Victor Hugo à ses concitoyens, distingue deux républiques, la « rouge » et la « tricolore », sans s'impliquer autrement que par l'affirmation de sa préférence pour la seconde; et lorsqu'il expose sa candidature devant les cinq associations d'art et d'industrie, ce n'est qu'en réponse à des interpellations qu'il accepte de sortir des considérations politiques pour parler un peu de lui - mais toujours en tant qu'orateur et écrivain, et toujours pour justifier ses intentions politiques par des écrits antérieurs ${ }^{18}$. Les candidatures imposent toutes une phase de légitimation et, chez les écrivains, celle-ci passe toujours par le rappel des écrits antérieurs, qu'ils soient politiques ou littéraires. Lorsque les écrivains justifient leur passé politique et idéologique, c'est toujours en l'adossant à des réalisations politiques (Lamartine) ou à l'ancienneté de principes largement énoncés dans l'œuvre littéraire (comme Hugo, qui rappelle aussi bien ses discours à la Chambre des pairs que Le Dernier Jour d'un condamné). De même, Vigny cite largement dans sa profession de foi

15. Dominique Dupart, op. cit., p. 12.

16. Alain Vaillant, « Avant-propos », Romantisme, no 135, op. cit., p. 3-8.

17. François Dubasque, «L'engagement politique d'Alfred de Vigny sous la II République », Annales de Bretagne et des pays de l'Ouest, $\mathrm{n}^{\circ}$ 115-1, 2008, p. 160.

18. Victor Hugo, «Séance des cinq associations », op. cit., p. 154-163. 
son discours de réception à l'Académie française du 29 janvier 1846. Jamais les écrivains ne s'écartent de ces deux thèmes : les intentions politiques et le passé littéraire; et même les amis qui soutiennent leurs campagnes par voie de presse osent à peine quelques excursions timides hors de ces terrains littéraire et politique strictement délimités.

Un exemple intéressant de l'absence des thèmes liés à l'intime dans les campagnes électorales est fourni par la série "Les Hommes de l'avenir » du quotidien La Liberté, journal des idées et des faits, dont l'un des principaux rédacteurs est Alexandre Dumas ${ }^{19}$. Les principaux écrivains candidats aux élections à l'Assemblée constituante (Lamartine, Dumas, puis Hugo) se voient consacrer plusieurs articles de cette série. Chacun consiste en un portrait en cinq à huit courts " chapitres » signés Paul Meurice (pour Dumas) ou Auguste Vacquerie (pour Hugo). Tous ont à peu près la même structure, et le même objectif, défini par Meurice et Vacquerie dans l'introduction à la première série, consacrée à Lamartine : « raconter [...] le passé des hommes de l'avenir et montrer les idées qui s'attachent, comme une traînée lumineuse, à chacun de ces hommes ${ }^{20} \gg$. Ainsi le portrait de Dumas (du 24 au 28 avril) le présente-t-il d'abord comme grand diffuseur d'idées, homme hardi et enthousiaste, compétent sur toutes les questions y compris agricoles, sans rien dire qui ne soit du domaine des compétences politiques; les seules qualités plus « personnelles » mises en avant relèvent de la sociabilité, et, donc, du registre public (et politique). En ce qui concerne Hugo, dont le portrait est tracé dans les livraisons du 20 au 22 avril, le premier chapitre souligne et prouve la cohérence de son action politique jusqu'en 1848, à travers l'examen de ses discours à la Chambre des pairs, relus dans la perspective des premières élections républicaines. Le deuxième chapitre est le récit de l'enfance de Victor Hugo, le plus «personnel»; mais l'unique sentiment décrit (le déchirement de l'enfant entre les sympathies politiques contradictoires de ses parents), vite évoqué, a pour seul but d'expliquer les « variations » politiques de Hugo, souvent dénoncées par ses contemporains. Les derniers chapitres démontrent les qualités intellectuelles hugoliennes qui sont traditionnellement celles du politique et de l'écrivain : " nature arrêtée et ferme », " combinaison de l'entraînement et du sangfroid », " un grand poète qui renferme un grand critique ${ }^{21}$ », et prouvent l'ancienneté de son amour du peuple. Bien que le portrait soit fait par un proche, dans un journal complaisant, et sans doute à la demande même de l'intéressé, ce n'est que l'homme public qui est décrit.

19. Ce quotidien à cinq centimes dont le rédacteur en chef est Lepoitevin-Saint-Alme, et les principaux rédacteurs Alexandre Dumas, Auguste Vitu et Arthur Ponroy, a connu un succès rapide, puisque deux mois après son premier numéro, le 2 mars 1848 , il paraît à 100000 exemplaires par jour.

20. La Liberté, journal des idées et des faits, 16 avril 1848.

21. Ibid. 


\section{Sue, Lamartine : le recours aux genres de l'écriture de soi}

On ne trouve pas davantage de traces de l'intime dans les écrits électoraux des autres écrivains. Dumas, dans son mensuel Le Mois, Lamartine, dans Le Conseiller du peuple, proposent des dissertations politiques, exclusivement; Eugène Sue, qui édite durant la campagne Le Républicain des campagnes, plaquette diffusée les dimanches dans les communes du Loiret, propose lui aussi un exposé exclusivement politique et idéologique (explications de ce qu'est le principe républicain, bienfaits de la liberté de la presse, du droit de réunion, du droit au travail, de l'association, etc.), et ne dit strictement rien de lui-même.

Toutefois, dans le texte de Sue, une particularité attire l'attention : si les thèmes abordés ne sont que politiques, la forme choisie et le ton qu'elle implique sont, eux, d'un tout autre registre. Il s'agit d' « entretiens » qui présentent l'allure familière de la causerie. Le locuteur semble s'adresser à des connaissances, à des amis de cœur (l'apostrophe « Mes amis et chers concitoyens » entame chaque causerie, et la première commence justement par « Je viens causer avec vous de nos affaires ${ }^{22}$ ", terme qui dénote à la fois la familiarité et le partage d'un même sociolecte rural). Ponctuellement, des intrusions du narrateur dans l'exposé des idées ramènent la sèche dissertation politique à un genre plus familier (« ̀̀ dimanche, mes amis; nous recauserons de ces trois mots inscrits sur le drapeau de la République : Liberté; Égalité; Fraternité ${ }^{3} \ldots$... ). On retrouve les mêmes emprunts aux genres littéraires de l'intime chez Lamartine, dans la Lettre aux dix départements parue en novembre 1848, mais qui consiste presque plus en une entreprise de justification qu'en une profession de foi électorale, puisqu'il s'agit pour Lamartine de justifier la disgrâce dans laquelle il est tombé après l'échec du gouvernement provisoire. Le genre choisi est celui de la lettre, genre littéraire de l'intime par excellence, et le ton adopté rappelle celui d'un autre genre de l'écriture de soi, la confession; la Lettre se distingue à ce titre de tous les autres textes électoraux que nous avons pu lire, pour rejoindre le ton des confessions littéraires : " Je vous dois compte de moi-même. Il ne faut pas que vous ayez à rougir quand on parlera de moi devant vous », annonce le malheureux héros de février. Et le vocabulaire employé dans plusieurs paragraphes est à l'avenant : « J'étais resté isolé, pensif, silencieux sur mon banc, contemplant cette catastrophe si soudaine, qu'elle ne laissait même pas le temps d'en mesurer la profondeur. Ému jusqu'à l'attendrissement par cette infortune [...], mon cœur luttait en moi contre la raison ${ }^{24}$... ». L'abandon des codes de 1'exercice électoral semble montrer qu'à la fin de l'été 1848, et bien avant son échec à l'élection

22. Eugène Sue, Le Républicain des campagnes, Paris, Imprimerie G. Gratiot, 1848, p. 3.

23. Ibid., p. 9.

24. Alphonse de Lamartine, Lettre aux dix départements, Paris, Michel Lévy Frères, 1848 (25 août), p. 3. 
présidentielle, Lamartine a déjà quitté la politique pour rejoindre la littérature, le vrai domaine de l'intime. Toujours est-il que si certains écrivains, très minoritaires, convoquent les genres littéraires de l'écriture du moi pour présenter leurs idées politiques, les thèmes abordés restent presque toujours exclusivement ceux réservés traditionnellement au domaine public.

\section{Vie politique, vie intérieure en 1848 : le refus des infractions}

Comment expliquer le caractère volontairement impersonnel des déclarations de foi des candidats aux élections de 1848? Les raisons en sont multiples, à la fois sociologiques, historiques ou littéraires. Le fait que la République soit dans sa prime jeunesse sous la Deuxième République est sans doute la première raison expliquant l'absence de recours à la sphère de l'intime dans les campagnes politiques. Comme l'analyse Michaël Fœssel dans La Privation de l'intime, c'est essentiellement le désintérêt des citoyens pour la politique qui amène les hommes politiques actuels à jouer "la proximité et l'humanité, comme des gages d'une identité reconquise entre les représentants et les citoyen ${ }^{25} \gg$. En 1848, ce désintérêt n'existe pas - bien au contraire, les nouveaux droits civiques passionnent et mobilisent les citoyens -, et la proximité entre les élus et les électeurs n'existe pas non plus, n'a jamais existé : les différences de classes sont telles, et si bien ancrées dans les modes de vie, que les citoyens n'ont pas encore ce désir d'identification - tout au plus un désir d'égalité en matière civique. Les hommes politiques n'ont donc pas à se raconter, mais à prouver leur valeur politique (le terme de « compétence » serait anachronique), et à la prouver exclusivement par leurs actes et leurs paroles publics. Il est vrai aussi qu'en 1848 , tout un travail de catéchèse républicaine (ou bonapartiste, ou monarchique!) est à faire, et que les idées, souvent neuves, multiples, en cours d'élaboration, occupent largement la scène politique - d'où la densité des tracts, brochures, articles politiques de la Deuxième République -, sans qu'il soit nécessaire de satisfaire d'autres curiosités chez l'électeur novice.

Ensuite, on peut invoquer la stricte séparation entre vie privée et vie publique, héritée de la Déclaration des droits de l'homme et du citoyen, qui garantit à chacun le droit à l'intimité de l'espace privé (article 18-1). De là le refus, unanimement partagé, de parasiter un domaine par l'autre. L'opinion, telle qu'elle s'exprime par la voix des écrivains, des publicistes et des critiques littéraires, souligne d'ailleurs à cette période l'altérité radicale des deux domaines et l'indécence qu'il y aurait à les mêler. Lorsqu'après l'échec de Lamartine à l'élection présidentielle les Confidences commencent à paraître dans La Presse en décembre 1848, Sainte-Beuve s'indigne de l'impudeur de cet étalage et reproche au poète de « couronner sa politique par des idylles », dénonçant « l'inconvenance d'une publication romanesque dans les circonstances graves où [Lamartine] s'est placé et où il a tout fait

25. Michaël Fœssel, op. cit., p. 10. 
pour placer son pays ${ }^{26} \gg$. Lamartine lui-même consacre quasiment l'intégralité de la lettre-préface à la justification de sa démarche de publication, et il déclare, parlant de ses propres sentiments et s'adressant à lui-même : « Ils seront moins à toi, quand ils seront à tout le monde ${ }^{27}$ » : ce n'est pas ici, comme dans notre conception moderne, le politique qui est dégradé par l'irruption de l'intime, mais bien l'intime qui est dégradé par la «publicité », c'est-à-dire par sa diffusion sur la scène publique. Ce qui choque, à cette époque, c'est ce qu'Aurélie Loiseleur a très justement appelé des « infractions ». Parlant du Lamartine des Confidences et des Nouvelles confidences, elle note : «Ses préfaces reflètent la conscience exacerbée d'une infraction. Les isotopes de l'exhibition, du viol, de la souillure tant physique que morale et du sacrilège [...] se trouvent repris sans relâche dans les premières pages des Nouvelles Confidences ${ }^{28} »$. C'est l'intimité qui est sacrifiée dans la collusion de la vie privée et de l'espace public, non l'inverse.

D'autre part, chez les poètes et écrivains romantiques, si l'intime désigne ce qui est le plus individuel et le plus profond (conformément à l'usage augustinien, l'intime a toujours pour eux une dimension superlative), le terme est également souvent associé à l'inexprimé, voire à l'inexprimable, au « gardé en soi ». Une recherche lexicologique des sens de l'adjectif « intime » dans l'œuvre hugolienne (correspondance comprise) montre que le terme est souvent associé aux amitiés les plus profondes, à l'amour, à des conversations absolument privées, lorsque ces sentiments et ces conversations dénotent le secret, la familiarité profonde, le refus de la « publicité ». Il semble dès lors que, chez Hugo du moins, il existe un partage assez net entre les opinions et les sentiments publics (ou publicisables) et une vérité du cœur qui doit échapper à la publicité. Sans doute est-ce pour cela que ses impressions et ses sentiments sur les événements et les personnels politiques contemporains sont réservés à des textes non publiés de son vivant (Choses vues, les « carnets », les lettres à la famille, tous documents plus tard réunis par ses éditeurs sous le titre « écrits intimes »). Comme si la sphère publique imposait une posture, exigeait que l'individu, et à plus forte raison le poète lyrique ou le romancier romantique, fît la preuve de sa capacité à s'abstraire des affections humaines pour prouver sa valeur politique.

Cette vision découle de la conception « classique »-gréco-romaine d'une séparation nécessaire entre sphère publique et sphère privée, où la contamination du public par le privé ne peut qu'entraîner le chaos,

26. Sainte-Beuve, «Les Confidences, par M. de Lamartine » (8 octobre 1849), Causeries du Lundi, Paris, Garnier frères, t. I, 1857 ( $3^{\mathrm{e}}$ éd.), p. 20.

27. Alphonse de Lamartine, Les Confidences, Paris, Michel Lévy, 1857, p. 2.

28. Aurélie Loiseleur, «Les Confidences, une médiation sur l'obscénité », dans Nicolas Courtinat (dir.), Lamartine : autobiographie, mémoires, fiction de soi, Clermont-Ferrand, Presses universitaires Blaise Pascal, 2009, p. 117. 
l'irruption des passions dans le domaine de la raison. « Pour les Athéniens, explique Michaël Fœssel, accéder au rang de citoyen libre impliquait de se rendre existentiellement disponible pour la délibération démocratique ${ }^{29}$. » Cette conception est ranimée par la Révolution française, qui redéfinit sphère publique et sphère privée en distinguant nettement les deux : «Rien de ce qui était particulier ne devait porter atteinte à la volonté générale de la nation nouvelle », explique Lynn Hunt ${ }^{30}$, car les intérêts privés sont suspects de porter atteinte à ceux du peuple. C'est exactement ce qui transparaît à la lecture des professions de foi des candidats aux élections de 1848, où la nécessité de constituer un nouvel espace public - l'espace démocratique semble imposer à chacun d'en extraire tout ce qui est proprement individuel. La chose publique, c'est la chose de tous - et non la chose de chacun. Elle impose de s'appliquer à l'intérêt général - thème récurrent des professions de foi de ces premières élections républicaines - en faisant abstraction des besoins et intérêts purement individuels. Et ceci apparaît tout particulièrement dans les professions de foi des républicains - ou du moins des candidats se réclamant tels. Si ce n'est pas le règlement des élections qui impose la décence, c'est bien le signe que celle-ci est intériorisée par tous les protagonistes de l'élection en 1848 : le privé, perçu comme le domaine des affects et des passions, n'a rien à faire dans la République, qui est une affaire de raison; d'autant plus que ces premières élections réellement démocratiques doivent prouver leur tenue et leur viabilité.

\section{Conclusion : élection et familiarité}

Ce n'est que vers la fin du siècle - les dernières années du Second Empire, la Troisième République -, que se développeront les biographies des grands hommes « intimes" (avec Le Biographe, Le Trombinoscope) et que la vie personnelle des hommes politiques viendra alimenter les campagnes politiques - mais dans des proportions qui n'ont aucune mesure avec ce qui se pratique un siècle plus tard. Victor Hugo, par exemple, usera volontiers de son statut d'aïeul et de grand-père, des douleurs de l'exil, du regret de la patrie, etc., pour se construire un personnage d'ancêtre rassurant pour sa candidature à l'Assemblée nationale, puis au Sénat. À quoi attribuer cette évolution? Sans doute à un certain apprentissage des règles de la vie politique en régime républicain, et à certaines vérités apprises durant la Deuxième République.

En effet, en 1848, ce sont les conceptions politiques, les principes républicains, monarchiques et impériaux qui s'affrontent et occupent tout le terrain des écrits politiques. Pourtant, lorsqu'on examine les résultats des élections à la Constituante, puis à la Législative, l'on peut s'interroger,

29. Michaël Fœssel, op. cit., p. 27.

30. Lynn Hunt, "Révolution française et vie privée », dans Philippe Ariès et Georges Duby (dir.), Histoire de la vie privée, vol. IV, Paris, Seuil, 1985, p. 19. 
malgré tout, sur l'impact des débats d'idées sur les choix des citoyens. Car après tout, qui est élu? Pas Vigny, ni même Dumas, dont les exposés idéologiques ont pu ennuyer. On constate que ce sont les candidats familiers aux électeurs qui sont élus : familiers car connus dans leur département et soutenus par les notables locaux - qui sont souvent, eux-mêmes, les candidats élus; familiers par leurs relations avec des personnages populaires et bien connus - ce qui vaut en grande partie à Louis-Napoléon Bonaparte son éclatant succès lors de l'élection présidentielle; familiers, aussi, parce que s'étant livrés, en amont et en parallèle de leur vie politique, dans des textes intimistes (poésie, autobiographie ou autres). Les poètes et écrivains élus - Lamartine, Hugo, Béranger, Lamennais - sont les plus célèbres, les plus populaires : ce sont des hommes qui étaient connus en dehors même de la vie politique; des hommes dont le public connaissait les états d'âme, la richesse intérieure, l'intimité, par d'autres biais : Les Rayons et les Ombres, Les Chants du Crépuscule, les Méditations poétiques... Bref, en 1848 comme aux $\mathrm{xx}^{\mathrm{e}}$ et $\mathrm{XxI}^{\mathrm{e}}$ siècles, on peut se demander si les élus ne le sont pas parce qu'ils paraissaient plus humains, plus proches, plus intimement connus que les autres. De là à conclure que les exposés politiques ont finalement un poids limité dans les régimes républicains et les sociétés médiatiques, c'est un pas que les politiciens et communicants d'aujourd'hui semblent parfois sur le point de franchir. 